\title{
A Modified Mixed Ishikawa Iteration for Common Fixed Points of Two Asymptotically Quasi Pseudocontractive Type Non-Self-Mappings
}

\author{
Yuanheng Wang and Huimin Shi \\ Department of Mathematics, Zhejiang Normal University, Zhejiang 321004, China \\ Correspondence should be addressed to Yuanheng Wang; wangyuanhengmath@163.com
}

Received 3 January 2014; Accepted 21 February 2014; Published 26 March 2014

Academic Editor: Rudong Chen

Copyright (C) 2014 Y. Wang and H. Shi. This is an open access article distributed under the Creative Commons Attribution License, which permits unrestricted use, distribution, and reproduction in any medium, provided the original work is properly cited.

A new modified mixed Ishikawa iterative sequence with error for common fixed points of two asymptotically quasi pseudocontractive type non-self-mappings is introduced. By the flexible use of the iterative scheme and a new lemma, some strong convergence theorems are proved under suitable conditions. The results in this paper improve and generalize some existing results.

\section{Introduction}

Let $E$ be a real Banach space with its dual $E^{*}$ and let $C$ be a nonempty, closed, and convex subset of $E$. The mapping $J$ : $E \rightarrow 2^{E^{*}}$ is the normalized duality mapping defined by

$$
\begin{array}{r}
J(x)=\left\{x^{*} \in E^{*}:\left\langle x, x^{*}\right\rangle=\|x\| \cdot\left\|x^{*}\right\|,\|x\|=\left\|x^{*}\right\|\right\}, \\
x \in E .
\end{array}
$$

Let $T: C \rightarrow E$ be a mapping. We denote the fixed point set of $T$ by $F(T)$; that is, $F(T)=\{x \in C: x=T x\}$. Recall that a mapping $T: C \rightarrow E$ is said to be nonexpansive if, for each $x, y \in C$,

$$
\|T x-T y\| \leq\|x-y\|
$$

$T$ is said to be asymptotically nonexpansive if there exists a sequence $k_{n} \subseteq[1, \infty)$ with $k_{n} \rightarrow 1$ as $n \rightarrow \infty$ such that

$$
\left\|T^{n} x-T^{n} y\right\| \leq k_{n}\|x-y\|, \quad \forall x, y \in C .
$$

A sequence of self-mappings $\left\{T_{i}\right\}_{i=1}^{\infty}$ on $C$ is said to be uniform Lipschitzian with the coefficient $L$ if, for any $i=1,2, \ldots$, the following holds:

$$
\left\|T_{i}^{n} x-T_{i}^{n} y\right\| \leq L\|x-y\|, \quad \forall x, y \in C .
$$

$T$ is said to be asymptotically pseudocontractive if there exist $k_{n} \subseteq[1, \infty)$ with $k_{n} \rightarrow 1$ as $n \rightarrow \infty$ and $j(x-y) \epsilon$ $J(x-y)$ such that

$$
\left\langle T^{n} x-T^{n} y, j(x-y)\right\rangle \leq k_{n}\|x-y\|^{2}, \quad \forall x, y \in C .
$$

It is obvious to see that every nonexpansive mapping is asymptotically nonexpansive and every asymptotically nonexpansive mapping is asymptotically pseudocontractive. Goebel and Kirk [1] introduced the class of asymptotically nonexpansive mappings in 1972. The class of asymptotically pseudocontractive mappings was introduced by Schu [2] and has been studied by various authors for its generalized mappings in Hilbert spaces, Banach spaces, or generalized topological vector spaces by using the modified Mann or Ishikawa iteration methods (see, e.g., [3-21]).

In 2003, Chidume et al. [22] studied fixed points of an asymptotically nonexpansive non-self-mapping $T: C \rightarrow$ $E$ and the strong convergence of an iterative sequence $\left\{x_{n}\right\}$ generated by

$$
x_{n+1}=P\left(\left(1-\alpha_{n}\right) x_{n}+\alpha_{n} T(P T)^{n-1} x_{n}\right), \quad n \geq 1, x_{1} \in C,
$$

where $P: E \rightarrow C$ is a nonexpansive retraction. 
In 2011, Zegeye et al. [23] proved a strong convergence of Ishikawa scheme to a uniformly L-Lipschitzian and asymptotically pseudocontractive mappings in the intermediate sense which satisfies the following inequality (see [24]):

$$
\limsup _{n \rightarrow \infty} \sup _{x, y \in C}\left(\left\langle T^{n} x-T^{n} y, x-y\right\rangle-k_{n}\|x-y\|^{2}\right) \leq 0,
$$$$
\forall x, y \in C,
$$

where $k_{n} \subseteq[1, \infty)$ with $k_{n} \rightarrow 1$ as $n \rightarrow \infty$.

Motivated and inspired by the above results, in this paper, we introduce a new modified mixed Ishikawa iterative sequence with error for common fixed points of two more generalized asymptotically quasi pseudocontractive type non-self-mappings. By the flexible use of the iterative scheme and a new lemma (i.e., Lemma 6 in this paper), under suitable conditions, we prove some strong convergence theorems. Our results extend and improve many results of other authors to a certain extent, such as $[6,8,14-23]$.

\section{Preliminaries}

Definition 1. Let $C$ be a nonempty closed convex subset of a real Banach space $E$. $C$ is said to be a nonexpansive retract (with $P$ ) of $E$ if there exists a nonexpansive mapping $P$ : $E \rightarrow C$ such that, for all $x \in C, P x=x$. And $P$ is called a nonexpansive retraction.

Let $T: C \rightarrow E$ be a non-self-mapping (maybe selfmapping). $T$ is called uniformly L-Lipschitzian (with $P$ ) if there exists a constant $L>0$ such that

$\left\|T(P T)^{n-1} x-T(P T)^{n-1} y\right\| \leq L\|x-y\|, \quad \forall x, y \in C, n \geq 1$.

$T$ is said to be asymptotically pseudocontractive (with $P$ ) if there exist $k_{n} \subseteq[1, \infty)$ with $k_{n} \rightarrow 1$ as $n \rightarrow \infty$ and $\forall x, y \in C, \exists j(x-y) \in J(x-y)$ such that

$$
\left\langle T(P T)^{n-1} x-T(P T)^{n-1} y, j(x-y)\right\rangle \leq k_{n}\|x-y\|^{2} .
$$

$T$ is said to be an asymptotically pseudocontractive type (with $P$ ) if there exist $k_{n} \subseteq[1, \infty)$ with $k_{n} \rightarrow 1$ as $n \rightarrow \infty$ and $\forall x, y \in C, j(x-y) \in J(x-y)$ such that

$$
\begin{array}{r}
\lim \sup _{n \rightarrow \infty} \sup _{x, y \in C} \liminf _{j(x-y) \in J(x-y)}\left(\left\langleT(P T)^{n-1} x-T(P T)^{n-1} y,\right.\right. \\
\left.j(x-y)\rangle-k_{n}\|x-y\|^{2}\right) \leq 0 .
\end{array}
$$

$T$ is said to be an asymptotically quasi pseudocontractive type (with $P$ ) if $F(T) \neq \emptyset$, for $p \in F(T)$, there exist $k_{n} \subseteq[1, \infty$ ) with $k_{n} \rightarrow 1$ as $n \rightarrow \infty$, and, $\forall x \in C, j(x-p) \in J(x-p)$ such that

$$
\begin{gathered}
\limsup _{n \rightarrow \infty} \sup _{x \in C} \liminf _{j(x-p) \in J(x-p)}\left(\left\langle T(P T)^{n-1} x-p, j(x-y)\right\rangle\right. \\
\left.-k_{n}\|x-p\|^{2}\right) \leq 0 .
\end{gathered}
$$

Remark 2. It is clear that every asymptotically pseudocontractive mapping (with $P$ ) is asymptotically pseudocontractive type (with $P$ ) and every asymptotically pseudocontractive type (with $P$ ) is asymptotically quasi pseudocontractive type (with $P$ ). If $T: C \rightarrow C$ is a self-mapping, then we can choose $P=I$ as the identical mapping and we can get the usual definition of asymptotically pseudocontractive mapping, and so forth.

Definition 3. Let $C$ be a nonexpansive retract (with $P$ ) of $E$, let $T_{1}, T_{2}: C \rightarrow E$ be two uniformly L-Lipschitzian non-self-mappings and let $T_{1}$ be an asymptotically quasi pseudocontractive type (with $P$ ).

The sequence $\left\{x_{n}\right\}$ is called the new modified mixed Ishikawa iterative sequence with error (with $P$ ), if $\left\{x_{n}\right\}$ is generated by

$$
\begin{aligned}
x_{n+1}=P & \left(1-\alpha_{n}-\gamma_{n}\right) x_{n}+\alpha_{n} T_{1}\left(P T_{1}\right)^{n-1} \\
& \left.\times\left(\left(1-\beta_{n}\right) y_{n}+\beta_{n} T_{1}\left(P T_{1}\right)^{n-1} y_{n}\right)+\gamma_{n} u_{n}\right), \\
y_{n}=P & \left(\left(1-\alpha_{n}^{\prime}-\gamma_{n}^{\prime}\right) x_{n}+\alpha_{n}^{\prime} T_{2}\left(P T_{2}\right)^{n-1}\right. \\
& \left.\times\left(\left(1-\beta_{n}^{\prime}\right) x_{n}+\beta_{n}^{\prime} T_{2}\left(P T_{2}\right)^{n-1} x_{n}\right)+\gamma_{n}^{\prime} v_{n}\right),
\end{aligned}
$$

where $x_{1} \in C$ is arbitrary, $\left\{u_{n}\right\}$ and $\left\{v_{n}\right\} \subset C$ are bounded, and $\alpha_{n}, \beta_{n}, \gamma_{n}, \alpha_{n}^{\prime}, \beta_{n}^{\prime}, \gamma_{n}^{\prime} \in[0,1], n=1,2, \ldots$

$$
\begin{aligned}
& \text { If } \alpha_{n}^{\prime}=\beta_{n}^{\prime}=\gamma_{n}^{\prime}=0,(12) \text { turns to } \\
& x_{n+1}=P\left(\left(1-\alpha_{n}-\gamma_{n}\right) x_{n}+\alpha_{n} T_{1}\left(P T_{1}\right)^{n-1}\right. \\
&\left.\times\left(\left(1-\beta_{n}\right) x_{n}+\beta_{n} T_{1}\left(P T_{1}\right)^{n-1} x_{n}\right)+\gamma_{n} u_{n}\right),
\end{aligned}
$$

and it is called the new modified mixed Mann iterative sequence with error (with $P$ ).

$$
\begin{aligned}
& \text { If } \gamma_{n}=\gamma_{n}^{\prime}=0,(12) \text { becomes } \\
& \begin{aligned}
x_{n+1}=P & \left(\left(1-\alpha_{n}\right) x_{n}+\alpha_{n} T_{1}\left(P T_{1}\right)^{n-1}\right. \\
& \left.\times\left(\left(1-\beta_{n}\right) y_{n}+\beta_{n} T_{1}\left(P T_{1}\right)^{n-1} y_{n}\right)\right), \\
y_{n}=P( & \left(1-\alpha_{n}^{\prime}\right) x_{n}+\alpha_{n}^{\prime} T_{2}\left(P T_{2}\right)^{n-1} \\
& \left.\times\left(\left(1-\beta_{n}^{\prime}\right) x_{n}+\beta_{n}^{\prime} T_{2}\left(P T_{2}\right)^{n-1} x_{n}\right)\right),
\end{aligned}
\end{aligned}
$$

and it is called the new modified mixed Ishikawa iterative sequence (with $P$ ).

If $\beta_{n}=\beta_{n}^{\prime}=0$, (14) turns to

$$
\begin{aligned}
x_{n+1} & =P\left(\left(1-\alpha_{n}\right) x_{n}+\alpha_{n} T_{1}\left(P T_{1}\right)^{n-1} y_{n}\right), \\
y_{n} & =P\left(\left(1-\alpha_{n}^{\prime}\right) x_{n}+\alpha_{n}^{\prime} T_{2}\left(P T_{2}\right)^{n-1} x_{n}\right),
\end{aligned}
$$

and it is called the new mixed Ishikawa iterative sequence (with $P$ ).

If $T_{1}=T_{2}=T: C \rightarrow C$ is a self-mapping and $P=I$ is the identical mapping, then (15) is just the modified Ishikawa iterative sequence

$$
\begin{aligned}
x_{n+1} & =\left(1-\alpha_{n}\right) x_{n}+\alpha_{n} T^{n} y_{n}, \\
y_{n} & =\left(1-\alpha_{n}^{\prime}\right) x_{n}+\alpha_{n}^{\prime} T^{n} x_{n} .
\end{aligned}
$$


If $\alpha_{n}^{\prime}=0$, (15) becomes (6), obviously. So, iterative method (12) is greatly generalized.

The following lemmas will be needed in what follows to prove our main results.

Lemma 4 (see [19]). Let $E$ be a real Banach space. Then, for all $x, y \in E, j(x+y) \in J(x+y)$, the following inequality holds:

$$
\|x+y\|^{2} \leq\|x\|^{2}+2\langle x, j(x+y)\rangle .
$$

Lemma 5 (see $[6,7]$ ). Let $\left\{a_{n}\right\},\left\{b_{n}\right\},\left\{c_{n}\right\}$ be three sequences of nonnegative numbers satisfying the recursive inequality:

$$
a_{n+1} \leq\left(1+b_{n}\right) a_{n}+c_{n}, \quad \forall n \geq n_{0}
$$

where $n_{0}$ is some nonnegative integer. If $\sum_{n=1}^{\infty} b_{n}<\infty, \Sigma_{n=1}^{\infty} c_{n}<$ $\infty$, then $\lim _{n \rightarrow \infty} a_{n}$ exists.

Lemma 6. Suppose that $\phi:[0,+\infty) \rightarrow[0,+\infty)$ is a strictly increasing function with $\phi(0)=0$. Let $\left\{a_{n}\right\},\left\{b_{n}\right\},\left\{c_{n}\right\},\left\{\lambda_{n}\right\}(0 \leq$ $\left.\lambda_{n} \leq 1\right)$ be four sequences of nonnegative numbers satisfying the recursive inequality:

$$
a_{n+1} \leq\left(1+b_{n}\right) a_{n}-\lambda_{n} \phi\left(a_{n+1}\right)+c_{n}, \quad \forall n \geq n_{0},
$$

where $n_{0}$ is some nonnegative integer. If $\sum_{n=1}^{\infty} b_{n}<\infty, \sum_{n=1}^{\infty} c_{n}<$ $\infty, \sum_{n=1}^{\infty} \lambda_{n}=\infty$, then $\lim _{n \rightarrow \infty} a_{n}=0$.

Proof. From (19), we get

$$
a_{n+1} \leq\left(1+b_{n}\right) a_{n}+c_{n}, \quad \forall n \geq n_{0} .
$$

By Lemma 5, we know that $\lim _{n \rightarrow \infty} a_{n}=a \geq 0$ exists. Let $M=\sup _{1 \leq n \leq \infty}\left\{a_{n}\right\}<\infty$. Now we show $a=0$. Otherwise, if $a>0$, then $\exists n_{1} \geq n_{0}$, such that $a_{n+1} \geq(1 / 2) a>0$ when $n \geq$ $n_{1}$. Because $\phi$ is a strictly increasing function, so $\phi\left(a_{n+1}\right) \geq$ $\phi((1 / 2) a)>0$. From (19) again, we have

$$
\begin{aligned}
0< & \phi\left(\frac{1}{2} a\right) \sum_{n=1}^{\infty} \lambda_{n} \\
= & \phi\left(\frac{1}{2} a\right) \sum_{n=1}^{n_{1}} \lambda_{n}+\phi\left(\frac{1}{2} a\right) \sum_{n=n_{1}+1}^{\infty} \lambda_{n} \\
\leq & \phi\left(\frac{1}{2} a\right) \sum_{n=1}^{n_{1}} \lambda_{n}+\sum_{n=n_{1}+1}^{\infty} \lambda_{n} \phi\left(a_{n+1}\right) \\
\leq & \phi\left(\frac{1}{2} a\right) \sum_{n=1}^{n_{1}} \lambda_{n}+\sum_{n=n_{1}+1}^{\infty}\left(a_{n}-a_{n+1}\right) \\
& +\sum_{n=n_{1}+1}^{\infty} b_{n} a_{n}+\sum_{n=n_{1}+1}^{\infty} c_{n} \\
\leq & \phi\left(\frac{1}{2} a\right) \sum_{n=1}^{n_{1}} \lambda_{n}+a_{n_{1}+1}+M \sum_{n=1}^{\infty} b_{n}+\sum_{n=1}^{\infty} c_{n}<\infty
\end{aligned}
$$

This is a contradiction with the given condition $\sum_{n=1}^{\infty} \lambda_{n}=\infty$. Therefore $\lim _{n \rightarrow \infty} a_{n}=0$.
Lemma 7. Suppose that $\phi:[0,+\infty) \rightarrow[0,+\infty)$ is a strictly increasing function with $\phi(0)=0$. Let $\left\{a_{n}\right\},\left\{b_{n}\right\},\left\{c_{n}\right\},\left\{\lambda_{n}\right\}(0 \leq$ $\left.\lambda_{n} \leq 1\right),\left\{\varepsilon_{n}\right\}$ be five sequences of nonnegative numbers satisfying the recursive inequality:

$$
a_{n+1} \leq\left(1+b_{n}\right) a_{n}-\lambda_{n} \phi\left(a_{n+1}\right)+c_{n}+\lambda_{n} \varepsilon_{n}, \quad \forall n \geq n_{0},
$$

where $n_{0}$ is some nonnegative integer. If $\Sigma_{n=1}^{\infty} b_{n}<\infty, \sum_{n=1}^{\infty} c_{n}<$ $\infty, \Sigma_{n=1}^{\infty} \lambda_{n}=\infty, \lim _{n \rightarrow \infty} \varepsilon_{n}=0$, then $\lim _{n \rightarrow \infty} a_{n}=0$.

Proof. Firstly, we show $\liminf _{n \rightarrow \infty} a_{n}=a=0$. If $a>0$, then, for arbitrary $r \in(0, a), \exists n_{1} \geq n_{0}$, such that $a_{n+1} \geq r>0$ when $n \geq n_{1}$. Because $\phi$ is a strictly increasing function and $\lim _{n \rightarrow \infty} \varepsilon_{n}=0$, so $\phi\left(a_{n+1}\right) \geq \phi(r)>0$ and $\varepsilon_{n} \leq(1 / 2) \phi(r)$ when $n \geq n_{1}$. From (22), we have

$$
\begin{aligned}
a_{n+1} & \leq\left(1+b_{n}\right) a_{n}-\lambda_{n} \phi\left(a_{n+1}\right)+c_{n}+\lambda_{n} \frac{1}{2} \phi\left(a_{n+1}\right) \\
& =\left(1+b_{n}\right) a_{n}-\frac{1}{2} \lambda_{n} \phi\left(a_{n+1}\right)+c_{n}, \quad \forall n \geq n_{1} .
\end{aligned}
$$

By Lemma 6, we get $0=\lim _{n \rightarrow \infty} a_{n}=\liminf _{n \rightarrow \infty} a_{n}=a>0$. This is contradictory. So, $\liminf _{n \rightarrow \infty} a_{n}=0$.

Secondly, $\forall \varepsilon>0$, from the given conditions in Lemma 7, $\exists n_{2} \geq n_{0}$, when $\forall n \geq n_{2}$, we have

$$
\varepsilon_{n} \leq \phi(\varepsilon), \quad \sum_{n=n_{2}}^{\infty} b_{n} \leq \ln 2, \quad \sum_{n=n_{2}}^{\infty} c_{n} \leq \varepsilon .
$$

On the other hand, since $\liminf _{n \rightarrow \infty} a_{n}=0, \exists N \geq n_{2}$ such that $a_{N} \leq \varepsilon$. Now we claim

$$
a_{k} \leq\left(\varepsilon+\sum_{n=N}^{k-1} c_{n}\right) \exp \left(\sum_{n=N}^{k-1} b_{n}\right), \quad \forall k \geq N
$$

In fact, when $k=N$, (25) holds. Suppose that (25) holds for $k$ dose not for $k+1$. Then

$$
a_{k+1}>\left(\varepsilon+\sum_{n=N}^{k} c_{n}\right) \exp \left(\sum_{n=N}^{k} b_{n}\right) .
$$

Furthermore, $a_{k+1}>\varepsilon, \phi\left(a_{k+1}\right)>\phi(\varepsilon)$. But by (22), (24), and the inductive hypothesis, we have

$$
\begin{aligned}
a_{n+1} & \leq\left(1+b_{n}\right) a_{n}-\lambda_{n} \phi\left(a_{n+1}\right)+c_{n}+\lambda_{n} \varepsilon_{n} \\
& \leq\left(1+b_{n}\right) a_{n}-\lambda_{n} \phi(\varepsilon)+c_{n}+\lambda_{n} \phi(\varepsilon) \\
& \leq\left(1+b_{n}\right)\left(\varepsilon+\sum_{n=N}^{k-1} c_{n}\right) \exp \left(\sum_{n=N}^{k-1} b_{n}\right)+c_{n} \\
& \leq\left(\varepsilon+\sum_{n=N}^{k-1} c_{n}\right) \exp \left(\sum_{n=N}^{k} b_{n}\right)+c_{n} \\
& \leq\left(\varepsilon+\sum_{n=N}^{k} c_{n}\right) \exp \left(\sum_{n=N}^{k} b_{n}\right) .
\end{aligned}
$$


This is a contradiction with (26). So, (25) holds. Whereupon,

$$
\begin{aligned}
\limsup _{k \rightarrow \infty} a_{k} & \leq\left(\varepsilon+\sum_{n=N}^{\infty} c_{n}\right) \exp \left(\sum_{n=N}^{\infty} b_{n}\right) \\
& \leq 2(\varepsilon+\varepsilon)=4 \varepsilon .
\end{aligned}
$$

Therefore, $\lim \sup _{k \rightarrow \infty} a_{k}=0=\lim _{n \rightarrow \infty} a_{n}$.

\section{Main Results}

Now, we are in a position to state and prove the main results of this paper.

Theorem 8. Let $C$ be nonexpansive retract (with $P$ ) of $a$ real Banach space E. Assume that $T_{1}, T_{2}: C \rightarrow E$ are two uniformly L-Lipschitzian non-self-mappings (with $P$ ) and $T_{1}$ is an asymptotically quasi pseudocontractive type with coefficient numbers $\left\{k_{n}\right\} \subset[1,+\infty): k_{n} \rightarrow 1$ satisfying $F=F\left(T_{1}\right) \cap F\left(T_{2}\right) \neq \emptyset$. Suppose that $\left\{u_{n}\right\},\left\{v_{n}\right\} \subset C$ are two bounded sequences; $\left\{\alpha_{n}\right\},\left\{\beta_{n}\right\},\left\{\gamma_{n}\right\},\left\{\alpha_{n}^{\prime}\right\},\left\{\beta_{n}^{\prime}\right\},\left\{\gamma_{n}^{\prime}\right\} \subset[0,1]$ are six number sequences satisfying the following:

$$
\begin{aligned}
& \text { (C1) } \sum_{n=1}^{\infty} \alpha_{n}=+\infty, \sum_{n=1}^{\infty} \alpha_{n}^{2}<+\infty, \sum_{n=1}^{\infty} \alpha_{n}\left(k_{n}-1\right)<+\infty ; \\
& \text { (C2) } \alpha_{n}+\gamma_{n} \leq 1, \alpha_{n}^{\prime}+\gamma_{n}^{\prime} \leq 1, \sum_{n=1}^{\infty} \gamma_{n}<+\infty ; \\
& \text { (C3) } \sum_{n=1}^{\infty} \alpha_{n} \beta_{n}<+\infty, \sum_{n=1}^{\infty} \alpha_{n} \alpha_{n}^{\prime}<+\infty, \sum_{n=1}^{\infty} \alpha_{n} \gamma_{n}^{\prime}<+\infty .
\end{aligned}
$$

If $x_{1} \in C$ is arbitrary, then the iterative sequence $\left\{x_{n}\right\}$ generated by (12) converges strongly to the fixed point $x^{*} \in F$ if and only if there exists a strictly increasing function $\phi:[0,+\infty) \rightarrow$ $[0,+\infty)$ with $\phi(0)=0$ such that

$$
\begin{aligned}
& \limsup _{n \rightarrow \infty} \inf _{j\left(x_{n+1}-x^{*}\right) \in J\left(x_{n+1}-x^{*}\right)}\left[\left\langleT_{1}\left(P T_{1}\right)^{n-1} x_{n+1}-x^{*},\right.\right. \\
&\left.j\left(x_{n+1}-x^{*}\right)\right\rangle-k_{n}\left\|x_{n+1}-x^{*}\right\|^{2} \\
&\left.+\phi\left(\left\|x_{n+1}-x^{*}\right\|\right)\right] \leq 0 .
\end{aligned}
$$

Proof. (Adequacy). Let

$$
\begin{gathered}
\varepsilon_{n}^{\prime}=\inf _{j\left(x_{n+1}-x^{*}\right) \in J\left(x_{n+1}-x^{*}\right)}\left[\left\langleT_{1}\left(P T_{1}\right)^{n-1} x_{n+1}-x^{*},\right.\right. \\
\left.\quad j\left(x_{n+1}-x^{*}\right)\right\rangle-k_{n}\left\|x_{n+1}-x^{*}\right\|^{2} \\
\left.+\phi\left(\left\|x_{n+1}-x^{*}\right\|\right)\right], \\
\varepsilon_{n}=\max \left\{\varepsilon_{n}^{\prime}, 0\right\}+\frac{1}{n} .
\end{gathered}
$$

Then there exists $j\left(x_{n+1}-x^{*}\right) \in J\left(x_{n+1}-x^{*}\right)$ such that

$$
\begin{aligned}
& \left\langle T_{1}\left(P T_{1}\right)^{n-1} x_{n+1}-x^{*}, j\left(x_{n+1}-x^{*}\right)\right\rangle \\
& \quad-k_{n}\left\|x_{n+1}-x^{*}\right\|^{2}+\phi\left(\left\|x_{n+1}-x^{*}\right\|\right) \leq \varepsilon_{n} .
\end{aligned}
$$

From (29), we know that $\lim \sup _{n \rightarrow \infty} \varepsilon_{n}^{\prime} \leq 0$. So, $\lim _{n \rightarrow \infty} \varepsilon_{n}=$ 0 .

Now, from the given conditions and (12), we can let

$$
\begin{aligned}
& \sigma_{n}=\left(1-\beta_{n}\right) y_{n}+\beta_{n} T_{1}\left(P T_{1}\right)^{n-1} y_{n}, \\
& \delta_{n}=\left(1-\beta_{n}^{\prime}\right) x_{n}+\beta_{n}^{\prime} T_{2}\left(P T_{2}\right)^{n-1} x_{n},
\end{aligned}
$$

and $M=\sup _{n \geq 1}\left\{\left\|\mu_{n}-x^{*}\right\|,\left\|\nu_{n}-x^{*}\right\|\right\}<\infty$. Then

$$
\begin{aligned}
& \left\|\delta_{n}-x^{*}\right\| \leq \beta_{n}^{\prime}\left\|T_{2}\left(P T_{2}\right) x_{n}-x^{*}\right\|+\left(1-\beta_{n}^{\prime}\right)\left\|x_{n}-x^{*}\right\| \\
& \leq \beta_{n}^{\prime} L\left\|x_{n}-x^{*}\right\|+\left\|x_{n}-x^{*}\right\| \\
& \left\|y_{n}-x^{*}\right\| \leq\left(1-\alpha_{n}^{\prime}-\gamma_{n}^{\prime}\right)\left\|x_{n}-x^{*}\right\| \\
& +\alpha_{n}^{\prime} L\left\|\delta_{n}-x^{*}\right\|+\gamma_{n}^{\prime}\left\|v_{n}-x^{*}\right\| \\
& \leq\left\|x_{n}-x^{*}\right\|+\alpha_{n}^{\prime} \beta_{n}^{\prime} L^{2}\left\|x_{n}-x^{*}\right\| \\
& +\alpha_{n}^{\prime} L\left\|x_{n}-x^{*}\right\|+\gamma_{n}^{\prime} M \\
& =\left(1+\alpha_{n}^{\prime} \beta_{n}^{\prime} L^{2}+\alpha_{n}^{\prime} L\right)\left\|x_{n}-x^{*}\right\|+\gamma_{n}^{\prime} M \\
& \leq\left(1+L+L^{2}\right)\left\|x_{n}-x^{*}\right\|+M \\
& \left\|\sigma_{n}-x^{*}\right\| \leq \beta_{n}\left\|T_{1}\left(P T_{1}\right)^{n-1} y_{n}-x^{*}\right\| \\
& +\left(1-\beta_{n}\right)\left\|y_{n}-x^{*}\right\| \\
& \leq \beta_{n} L\left\|y_{n}-x^{*}\right\|+\left\|y_{n}-x^{*}\right\| \\
& \leq(1+L)\left(1+L+L^{2}\right)\left\|x_{n}-x^{*}\right\|+(1+L) M ; \\
& \left\|y_{n}-x_{n+1}\right\| \leq \alpha_{n} L\left\|\sigma_{n}-x^{*}\right\|+\alpha_{n}\left\|x_{n}-x^{*}\right\| \\
& +\alpha_{n}^{\prime} L\left\|\delta_{n}-x^{*}\right\|+\alpha_{n}^{\prime}\left\|x_{n}-x^{*}\right\| \\
& +\left(\gamma_{n}+\gamma_{n}^{\prime}\right)\left\|x_{n}-x^{*}\right\|+\left(\gamma_{n}+\gamma_{n}^{\prime}\right) M \\
& \leq \alpha_{n} L\left[(1+L)\left(1+L+L^{2}\right)\left\|x_{n}-x^{*}\right\|\right. \\
& +(1+L) M] \\
& +\alpha_{n}^{\prime} L\left[\left(1+\beta_{n}^{\prime} L\right)\left\|x_{n}-x^{*}\right\|\right] \\
& +\left(\alpha_{n}+\alpha_{n}^{\prime}+\gamma_{n}+\gamma_{n}^{\prime}\right)\left\|x_{n}-x^{*}\right\|+\left(\gamma_{n}+\gamma_{n}^{\prime}\right) M \\
& \leq\left[\alpha_{n} L(1+L)\left(1+L+L^{2}\right)+\alpha_{n}^{\prime} L\left(1+\beta_{n}^{\prime} L\right)\right. \\
& \left.+\alpha_{n}+\alpha_{n}^{\prime}+\gamma_{n}+\gamma_{n}^{\prime}\right]\left\|x_{n}-x^{*}\right\| \\
& +\left(\alpha_{n} L(1+L)+\gamma_{n}+\gamma_{n}^{\prime}\right) M
\end{aligned}
$$

$$
\begin{aligned}
\left\|\sigma_{n}-x_{n+1}\right\| & \leq\left\|y_{n}-x_{n+1}\right\|+\beta_{n}\left\|T_{1}\left(P T_{1}\right)^{n-1} y_{n}-y_{n}\right\| \\
& \leq s_{n}\left\|x_{n}-x^{*}\right\|+t_{n}
\end{aligned}
$$


where

$$
\begin{gathered}
s_{n}=\alpha_{n} L(1+L)\left(1+L+L^{2}\right)+\alpha_{n}^{\prime} L\left(1+\beta_{n}^{\prime} L\right)+\alpha_{n} \\
+\alpha_{n}^{\prime}+\gamma_{n}+\gamma_{n}^{\prime}+\beta_{n}(1+L)\left(1+L+L^{2}\right) ; \\
t_{n}=\left[\alpha_{n} L(1+L)+\gamma_{n}+\gamma_{n}^{\prime}+\beta_{n}(1+L)\right] M .
\end{gathered}
$$

So, by Lemma 4 ,

$$
\begin{aligned}
2 \alpha_{n} & \left\langle T_{1}\left(P T_{1}\right)^{n-1} \sigma_{n}-T_{1}\left(P T_{1}\right)^{n-1} x_{n+1}, j\left(x_{n+1}-x^{*}\right)\right\rangle \\
\leq & 2 \alpha_{n} L\left\|x_{n+1}-x^{*}\right\|\left\|\sigma_{n}-x_{n+1}\right\| \\
\leq & 2 \alpha_{n} L\left\|x_{n+1}-x^{*}\right\|\left[s_{n}\left\|x_{n}-x^{*}\right\|+t_{n}\right] ; \\
\left\|x_{n+1}-x^{*}\right\|^{2} & \\
\leq & \left(1-\alpha_{n}-\gamma_{n}\right)^{2}\left\|x_{n}-x^{*}\right\|^{2} \\
& +2 \alpha_{n}\left\langle T_{1}\left(P T_{1}\right)^{n-1} \sigma_{n}-x^{*}, j\left(x_{n+1}-x^{*}\right)\right\rangle \\
& +2 \gamma_{n}\left\langle\mu_{n}-x^{*}, j\left(x_{n+1}-x^{*}\right)\right\rangle \\
\leq & \left(1-\alpha_{n}-\gamma_{n}\right)^{2}\left\|x_{n}-x^{*}\right\|^{2} \\
& +2 \alpha_{n}\left\langle T_{1}\left(P T_{1}\right)^{n-1} \sigma_{n}-T_{1}\left(P T_{1}\right)^{n-1} x_{n+1},\right. \\
\quad & \left.\quad j\left(x_{n+1}-x^{*}\right)\right\rangle \\
& +2 \alpha_{n}\left\langle T_{1}\left(P T_{1}\right)^{n-1} x_{n+1}-x^{*}, j\left(x_{n+1}-x^{*}\right)\right\rangle \\
& +2 \gamma_{n} M\left\|x_{n+1}-x^{*}\right\| .
\end{aligned}
$$

For the third in (36), we have

$$
\begin{aligned}
2 \alpha_{n} & \left\langle T_{1}\left(P T_{1}\right)^{n-1} x_{n+1}-x^{*}, j\left(x_{n+1}-x^{*}\right)\right\rangle \\
& =2 \alpha_{n} d_{n}+2 \alpha_{n}\left[k_{n}\left\|x_{n+1}-x^{*}\right\|^{2}-\phi\left(\left\|x_{n+1}-x^{*}\right\|\right)\right] \\
& \leq 2 \alpha_{n} \varepsilon_{n}+2 \alpha_{n}\left[k_{n}\left\|x_{n+1}-x^{*}\right\|^{2}-\phi\left(\left\|x_{n+1}-x^{*}\right\|\right)\right],
\end{aligned}
$$

where

$$
\begin{aligned}
d_{n}= & \left\langle T_{1}\left(P T_{1}\right)^{n-1} x_{n+1}-x^{*}, j\left(x_{n+1}-x^{*}\right)\right\rangle \\
& -k_{n}\left\|x_{n+1}-x^{*}\right\|^{2}+\phi\left(\left\|x_{n+1}-x^{*}\right\|\right) \leq \varepsilon_{n} .
\end{aligned}
$$

Substituting (35) into (36), we get

$$
\begin{aligned}
\left\|x_{n+1}-x^{*}\right\|^{2} \leq & \left(1-\alpha_{n}\right)^{2}\left\|x_{n}-x^{*}\right\|^{2}+2 \alpha_{n} \varepsilon_{n} \\
& +2 \alpha_{n} k_{n}\left\|x_{n+1}-x^{*}\right\|^{2}-2 \alpha_{n} \phi\left(\left\|x_{n+1}-x^{*}\right\|\right) \\
& +2 \alpha_{n} L\left(s_{n}\left\|x_{n}-x^{*}\right\|+t_{n}\right)\left\|x_{n+1}-x^{*}\right\| \\
& +2 \gamma_{n} M\left\|x_{n+1}-x^{*}\right\| .
\end{aligned}
$$

Let $a_{n}=\left\|x_{n}-x^{*}\right\|^{2}, \varphi(t)=2 \phi(\sqrt{t})$, and

$$
\begin{aligned}
\xi_{n}= & L \alpha_{n} s_{n} \\
= & L^{2} \alpha_{n}^{2}(1+L)\left(1+L+L^{2}\right) \\
& +\alpha_{n} \alpha_{n}^{\prime} L^{2}\left(1+\beta_{n}^{\prime} L\right)+\alpha_{n}^{2} L+\alpha_{n} \alpha_{n}^{\prime} L+L \alpha_{n} \gamma_{n} \\
& +L \alpha_{n} \gamma_{n}^{\prime}+L \alpha_{n} \beta_{n}(1+L)\left(1+L+L^{2}\right), \\
\rho_{n}= & L \alpha_{n} t_{n}+M \gamma_{n} \\
= & {\left[\alpha_{n}^{2} L^{2}(1+L)+L \alpha_{n} \gamma_{n}+L \alpha_{n} \gamma_{n}^{\prime}+\alpha_{n} \beta_{n}\left(L+L^{2}\right)\right] M } \\
& +\gamma_{n} M .
\end{aligned}
$$

Then (39) becomes

$$
\begin{aligned}
a_{n+1} \leq & \left(1-\alpha_{n}\right)^{2} a_{n}+2 \alpha_{n} \varepsilon_{n}+2 \alpha_{n} k_{n} a_{n+1}-\alpha_{n} \varphi\left(a_{n+1}\right) \\
& +2\left(\xi_{n}\left\|x_{n}-x^{*}\right\|+\rho_{n}\right)\left\|x_{n+1}-x^{*}\right\| .
\end{aligned}
$$

By using $2 a b \leq a^{2}+b^{2}$, we have

$$
\begin{aligned}
a_{n+1} \leq & \left(1-\alpha_{n}\right)^{2} a_{n}+2 \alpha_{n} \varepsilon_{n}+2 \alpha_{n} k_{n} a_{n+1} \\
& -\alpha_{n} \varphi\left(a_{n+1}\right)+\xi_{n}\left(a_{n}+a_{n+1}\right)+\rho_{n}\left(1+a_{n+1}\right) \\
= & \left(1-2 \alpha_{n}+\alpha_{n}^{2}+\xi_{n}\right) a_{n}+\left(2 \alpha_{n} k_{n}+\xi_{n}+\rho_{n}\right) a_{n+1} \\
& -\alpha_{n} \varphi\left(a_{n+1}\right)+2 \alpha_{n} \varepsilon_{n}+\rho_{n} .
\end{aligned}
$$

From (40), (41), and the given conditions, we know

$$
\sum_{n=1}^{\infty} \alpha_{n}^{2}<+\infty, \quad \sum_{n=1}^{\infty} \xi_{n}<+\infty, \quad \sum_{n=1}^{\infty} \rho_{n}<+\infty .
$$

Then, $\lim _{n \rightarrow \infty}\left(2 \alpha_{n} k_{n}+\xi_{n}+\rho_{n}\right)=0$. Therefore $\exists n_{0}$, when $n \geq n_{0}, 2 \alpha_{n} k_{n}+\xi_{n}+\rho_{n} \leq 1 / 2$. Let

$$
\begin{aligned}
& b_{n}=\frac{1-2 \alpha_{n}+\alpha_{n}^{2}+\xi_{n}}{1-2 \alpha_{n} k_{n}-\xi_{n}-\rho_{n}}-1=\frac{2 \alpha_{n}\left(k_{n}-1\right)+\alpha_{n}^{2}+2 \xi_{n}+\rho_{n}}{1-2 \alpha_{n} k_{n}-\xi_{n}-\rho_{n}} ; \\
& c_{n}=\frac{\rho_{n}}{1-2 \alpha_{n} k_{n}-\xi_{n}-\rho_{n}} .
\end{aligned}
$$

So, when $n \geq n_{0}$, we get

$$
0 \leq b_{n} \leq 2\left[2 \alpha_{n}\left(k_{n}-1\right)+\alpha_{n}^{2}+2 \xi_{n}+\rho_{n}\right], \quad 0 \leq c_{n} \leq 2 \rho_{n} .
$$

From (44) and the given conditions, we have $\sum_{n=n_{0}}^{\infty} b_{n}<+\infty$, $\sum_{n=n_{0}}^{\infty} c_{n}<+\infty$. On the other hand, from (43), we have

$$
a_{n+1} \leq\left(1+b_{n}\right) a_{n}-\alpha_{n} \varphi\left(a_{n+1}\right)+4 \alpha_{n} \varepsilon_{n}+c_{n}, \quad \forall n \geq n_{0} .
$$


By Lemma 7, we at last get

$$
\lim _{n \rightarrow \infty} a_{n}=\lim _{n \rightarrow \infty}\left\|x_{n}-x^{*}\right\|^{2}=0 ;
$$

for example, $\lim _{n \rightarrow \infty} x_{n}=x^{*} \in F=F\left(T_{1}\right) \cap F\left(T_{2}\right)$.

(Necessity). Suppose that $\lim _{n \rightarrow \infty} x_{n}=x^{*} \in F$. Then we can choose an arbitrary continuous strictly increasing function $\phi:[0,+\infty) \rightarrow[0,+\infty)$ with $\phi(0)=0$, such as $\phi(t)=t$. We can get $\lim _{n \rightarrow \infty} \phi\left(\left\|x_{n+1}-x^{*}\right\|\right)=0$.

Because $T_{1}$ is an asymptotically quasi pseudocontractive type (with $P$ ), by (11) in Definition 1 , for any $p \in F\left(T_{1}\right) \supseteq F$, we have

$$
\begin{gathered}
\limsup _{n \rightarrow \infty} \sup _{x \in C} \liminf _{j(x-p) \in J(x-p)}\left(\left\langle T(P T)^{n-1} x-p, j(x-y)\right\rangle\right. \\
\left.-k_{n}\|x-p\|^{2}\right) \leq 0 .
\end{gathered}
$$

So,

$$
\begin{gathered}
\limsup _{n \rightarrow \infty} \inf _{j\left(x_{n+1}-x^{*}\right) \in J\left(x_{n+1}-x^{*}\right)}\left[\left\langleT_{1}\left(P T_{1}\right)^{n-1} x_{n+1}-x^{*},\right.\right. \\
\left.j\left(x_{n+1}-x^{*}\right)\right\rangle-k_{n}\left\|x_{n+1}-x^{*}\right\|^{2} \\
\left.+\phi\left(\left\|x_{n+1}-x^{*}\right\|\right)\right] \\
\limsup _{n \rightarrow \infty} \inf _{j\left(x_{n+1}-x^{*}\right) \in J\left(x_{n+1}-x^{*}\right)}\left[\left\langleT_{1}\left(P T_{1}\right)^{n-1} x_{n+1}-x^{*},\right.\right. \\
\left.\quad j\left(x_{n+1}-x^{*}\right)\right\rangle \\
\left.-k_{n}\left\|x_{n+1}-x^{*}\right\|^{2}\right] \\
+\lim _{n \rightarrow \infty} \phi\left(\left\|x_{n+1}-x^{*}\right\|\right) \leq 0+0=0 ;
\end{gathered}
$$

that is, (29) holds. This completes the proof of Theorem 8.

Combining with Theorem 8 and Definition 3, we have some results as follows.

Theorem 9. Let $C$ be nonexpansive retract (with $P$ ) of a real Banach space $E$. Assume that $T_{1}, T_{2}: C \rightarrow E$ are two uniformly L-Lipschitzian non-self-mappings (with $P$ ) and $T_{1}$ is an asymptotically quasi pseudocontractive type with coefficient numbers $\left\{k_{n}\right\} \subset[1,+\infty): k_{n} \rightarrow 1$ satisfying $F=F\left(T_{1}\right) \cap$ $F\left(T_{2}\right) \neq \emptyset$. Suppose that $\left\{\alpha_{n}\right\},\left\{\beta_{n}\right\},\left\{\alpha_{n}^{\prime}\right\},\left\{\beta_{n}^{\prime}\right\} \subset[0,1]$ are four number sequences satisfying the following:

$$
\begin{aligned}
& \text { (C1) } \sum_{n=1}^{\infty} \alpha_{n}=+\infty, \sum_{n=1}^{\infty} \alpha_{n}^{2}<+\infty, \sum_{n=1}^{\infty} \alpha_{n}\left(k_{n}-1\right)<+\infty ; \\
& \text { (C2) } \sum_{n=1}^{\infty} \alpha_{n} \beta_{n}<+\infty, \sum_{n=1}^{\infty} \alpha_{n} \alpha_{n}^{\prime}<+\infty .
\end{aligned}
$$

If $x_{1} \in C$ is arbitrary, then the iterative sequence $\left\{x_{n}\right\}$ generated by (14) converges strongly to the fixed point $x^{*} \in F$ if and only if there exists a strictly increasing function $\phi:[0,+\infty) \rightarrow$ $[0,+\infty)$ with $\phi(0)=0$ such that (29) holds.

Theorem 10. Let $C$ be nonexpansive retract (with $P$ ) of a real Banach space $E$. Assume that $T_{1}, T_{2}: C \rightarrow E$ are two uniformly L-Lipschitzian non-self-mappings (with $P$ ) and $T_{1}$ is an asymptotically quasi pseudocontractive type with coefficient numbers $\left\{k_{n}\right\} \subset[1,+\infty): k_{n} \rightarrow 1$ satisfying $F=F\left(T_{1}\right) \cap$ $F\left(T_{2}\right) \neq \emptyset$. Suppose that $\left\{\alpha_{n}\right\},\left\{\alpha_{n}^{\prime}\right\} \subset[0,1]$ are two number sequences satisfying the following:

(C1) $\sum_{n=1}^{\infty} \alpha_{n}=+\infty, \sum_{n=1}^{\infty} \alpha_{n}^{2}<+\infty, \sum_{n=1}^{\infty} \alpha_{n}\left(k_{n}-1\right)<+\infty$;

(C2) $\sum_{n=1}^{\infty} \alpha_{n} \alpha_{n}^{\prime}<+\infty$.

If $x_{1} \in C$ is arbitrary, then the iterative sequence $\left\{x_{n}\right\}$ generated by (15) converges strongly to the fixed point $x^{*} \in F$ if and only if there exists a strictly increasing function $\phi:[0,+\infty) \rightarrow$ $[0,+\infty)$ with $\phi(0)=0$ such that $(29)$ holds.

Theorem 11. Let $C$ be a nonempty closed convex subset of a real Banach space E. Assume that $T: C \rightarrow C$ is uniformly L-Lipschitzian self-mappings and asymptotically quasi pseudocontractive type with coefficient numbers $\left\{k_{n}\right\} \subset[1,+\infty)$ : $k_{n} \rightarrow 1$ satisfying $F=F(T) \neq \emptyset$. Suppose that $\left\{\alpha_{n}\right\},\left\{\alpha_{n}^{\prime}\right\} \subset$ $[0,1]$ are two number sequences satisfying the following:

$$
\begin{aligned}
& \text { (C1) } \sum_{n=1}^{\infty} \alpha_{n}=+\infty, \sum_{n=1}^{\infty} \alpha_{n}^{2}<+\infty, \sum_{n=1}^{\infty} \alpha_{n}\left(k_{n}-1\right)<+\infty \text {; } \\
& \text { (C2) } \sum_{n=1}^{\infty} \alpha_{n} \alpha_{n}^{\prime}<+\infty .
\end{aligned}
$$

If $x_{1} \in C$ is arbitrary, then the iterative sequence $\left\{x_{n}\right\}$ generated by (16) converges strongly to the fixed point $x^{*} \in F$ if and only if there exists a strictly increasing function $\phi:[0,+\infty) \rightarrow$ $[0,+\infty)$ with $\phi(0)=0$ such that (29) holds.

Remark 12. Our research and results in this paper have the following several advantaged characteristics. (a) The iterative scheme is the new modified mixed Ishikawa iterative scheme with error on two mappings $T_{1}, T_{2}$. (b) The common fixed point $x^{*} \in F=F\left(T_{1}\right) \cap F\left(T_{2}\right)$ is studied. (c) The research object is the very generalized asymptotically quasi pseudocontractive type (with $P$ ) non-self-mapping. (d) The tool used by us is the very powerful tool: Lemma 7. So, our results here extend and improve many results of other authors to a certain extent, such as $[6,8,14-23]$, and the proof methods are very different from the previous.

\section{Conflict of Interests}

The authors declare that there is no conflict of interests regarding the publication of this paper.

\section{Acknowledgments}

The authors would like to thank the editors and referees for their many useful comments and suggestions for the improvement of the paper. This work was supported by the National Natural Science Foundations of China (Grant no. 11271330) and the Natural Science Foundations of Zhejiang Province (Grant no. Y6100696).

\section{References}

[1] K. Goebel and W. A. Kirk, "A fixed point theorem for asymptotically nonexpansive mappings," Proceedings of the American Mathematical Society, vol. 35, pp. 171-174, 1972. 
[2] J. Schu, "Approximation of fixed points of asymptotically nonexpansive mappings," Proceedings of the American Mathematical Society, vol. 112, pp. 143-151, 1991.

[3] W. Takahashi, N.-C. Wong, and J.-C. Yao, "Attractive point and weak convergence theorems for new generalized hybrid mappings in Hilbert spaces," Journal of Nonlinear and Convex Analysis, vol. 13, pp. 745-757, 2012.

[4] Y. Kurokawa and W. Takahashi, "Weak and strong convergence theorems for nonspreading mappings in Hilbert spaces," Nonlinear Analysis, Theory, Methods and Applications, vol. 73, no. 6, pp. 1562-1568, 2010.

[5] H. K. Xu, "Vistosity approximation methods for nonexpansive mappings," Journal of Mathematical Analysis and Applications, vol. 298, pp. 279-291, 2004.

[6] D. R. Sahu, H.-K. Xu, and J.-C. Yao, "Asymptotically strict pseudocontractive mappings in the intermediate sense," Nonlinear Analysis, Theory, Methods and Applications, vol. 70, no. 10, pp. 3502-3511, 2009.

[7] J. K. Kim, D. R. Sahu, and Y. M. Nam, "Convergence theorem for fixed points of nearly uniformly L-Lipschitzian asymptotically generalized $\Phi$-hemicontractive mappings," Nonlinear Analysis, Theory, Methods and Applications, vol. 71, no. 12, pp. e2833e2838, 2009.

[8] S. S. Chang, "Some results for asymptotically pseudo-contractive mappings and asymptotically nonexpansive mappings," Proceedings of the American Mathematical Society, vol. 129, no. 3, pp. 845-853, 2001.

[9] P. Cholamjiak and S. Suantai, "A new hybrid algorithm for variational inclusions, generalized equilibrium problems, and a finite family of quasi-nonexpansive mappings," Fixed Point Theory and Applications, vol. 2009, Article ID 350979, 7 pages, 2009.

[10] C. Klin-Eam and S. Suantai, "Strong convergence of composite iterative schemes for a countable family of nonexpansive mappings in Banach spaces," Nonlinear Analysis, Theory, Methods and Applications, vol. 73, no. 2, pp. 431-439, 2010.

[11] S. Plubtieng and K. Ungchittrakool, "Approximation of common fixed points for a countable family of relatively nonexpansive mappings in a Banach space and applications," Nonlinear Analysis, Theory, Methods and Applications, vol. 72, no. 6, pp. 2896-2908, 2009.

[12] T. Suzuki, "Strong convergence theorems for infinite families of nonexpansive mappings in general Banach spaces," Fixed Point Theory and Applications, vol. 2005, no. 1, pp. 103-123, 2005.

[13] L.-C. Zeng and J.-C. Yao, "Implicit iteration scheme with perturbed mapping for common fixed points of a finite family of nonexpansive mappings," Nonlinear Analysis, Theory, Methods and Applications, vol. 64, no. 11, pp. 2507-2515, 2006.

[14] G. Marino and H.-K. Xu, "A general iterative method for nonexpansive mappings in Hilbert spaces," Journal of Mathematical Analysis and Applications, vol. 318, no. 1, pp. 43-52, 2006.

[15] Y.-H. Wang and Y.-H. Xia, "Strong convergence for asymptotically pseudocontractions with the demiclosedness principle in Banach spaces," Fixed Point Theory and Applications, vol. 2012, 8 pages, 2012.

[16] Y.-H. Wang and W. F. Xuan, "Convergence theorems for common fixed points of a finite family of relatively nonexpansive mappings in Banach spaces," Abstract and Applied Analysis, vol. 2013, Article ID 259470, 7 pages, 2013.

[17] L.-C. Zeng and J.-C. Yao, "Stability of iterative procedures with errors for approximating common fixed points of a couple of q-contractive-like mappings in Banach spaces," Journal of Mathematical Analysis and Applications, vol. 321, no. 2, pp. 661674, 2006.

[18] L.-C. Zeng, T. Tanaka, and J.-C. Yao, "Iterative construction of fixed points of nonself-mappings in Banach spaces," Journal of Computational and Applied Mathematics, vol. 206, no. 2, pp. 814-825, 2007.

[19] L. C. Zeng, N. C. Wong, and J. C. Yao, "Convergence analysis of iterative sequences for a pair of mappings in Banach spaces," Acta Mathematica Sinica, vol. 24, no. 3, pp. 463-470, 2008.

[20] X. Wu, J. C. Yao, and L. C. Zeng, "Uniformly normal structure and strong convergence theorems for asymptotically pseudocontractive mappings," Journal of Nonlinear and Convex Analysis, vol. 6, no. 3, pp. 453-463, 2005.

[21] L.-C. Ceng, N.-C. Wong, and J.-C. Yao, "Fixed point solutions of variational inequalities for a finite family of asymptotically nonexpansive mappings without common fixed point assumption," Computers and Mathematics with Applications, vol. 56, no. 9, pp. 2312-2322, 2008.

[22] C. E. Chidume, E. U. Ofoedu, and H. Zegeye, "Strong and weak convergence theorems for asymptotically nonexpansive mappings," Journal of Mathematical Analysis and Applications, vol. 280, no. 2, pp. 364-374, 2003.

[23] H. Zegeye, M. Robdera, and B. Choudhary, "Convergence theorems for asymptotically pseudocontractive mappings in the intermediate sense," Computers and Mathematics with Applications, vol. 62, no. 1, pp. 326-332, 2011.

[24] S.-S. Chang, "Some problems and results in the study of nonlinear analysis," Nonlinear Analysis, Theory, Methods and Applications, vol. 30, no. 7, pp. 4197-4208, 1997. 


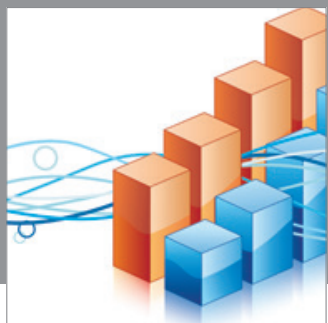

Advances in

Operations Research

mansans

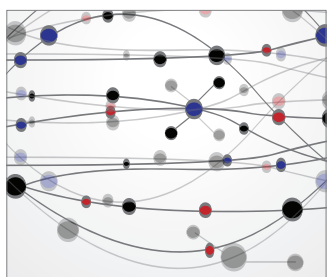

The Scientific World Journal
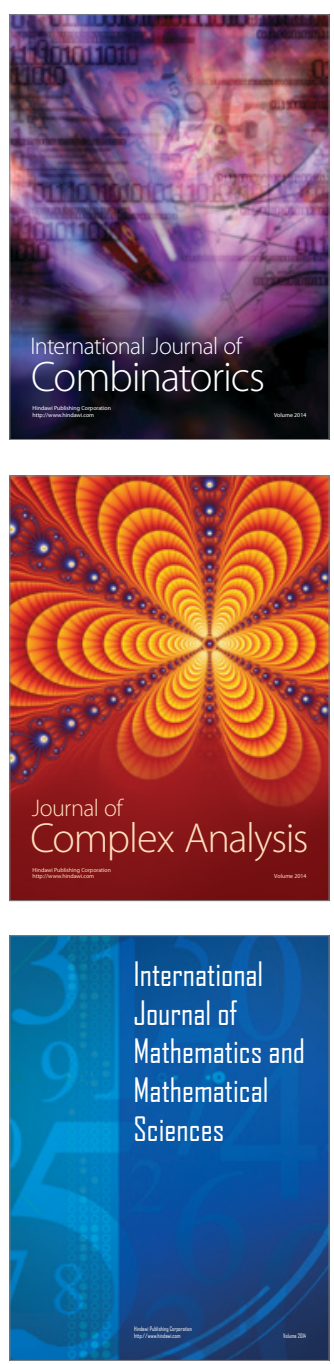
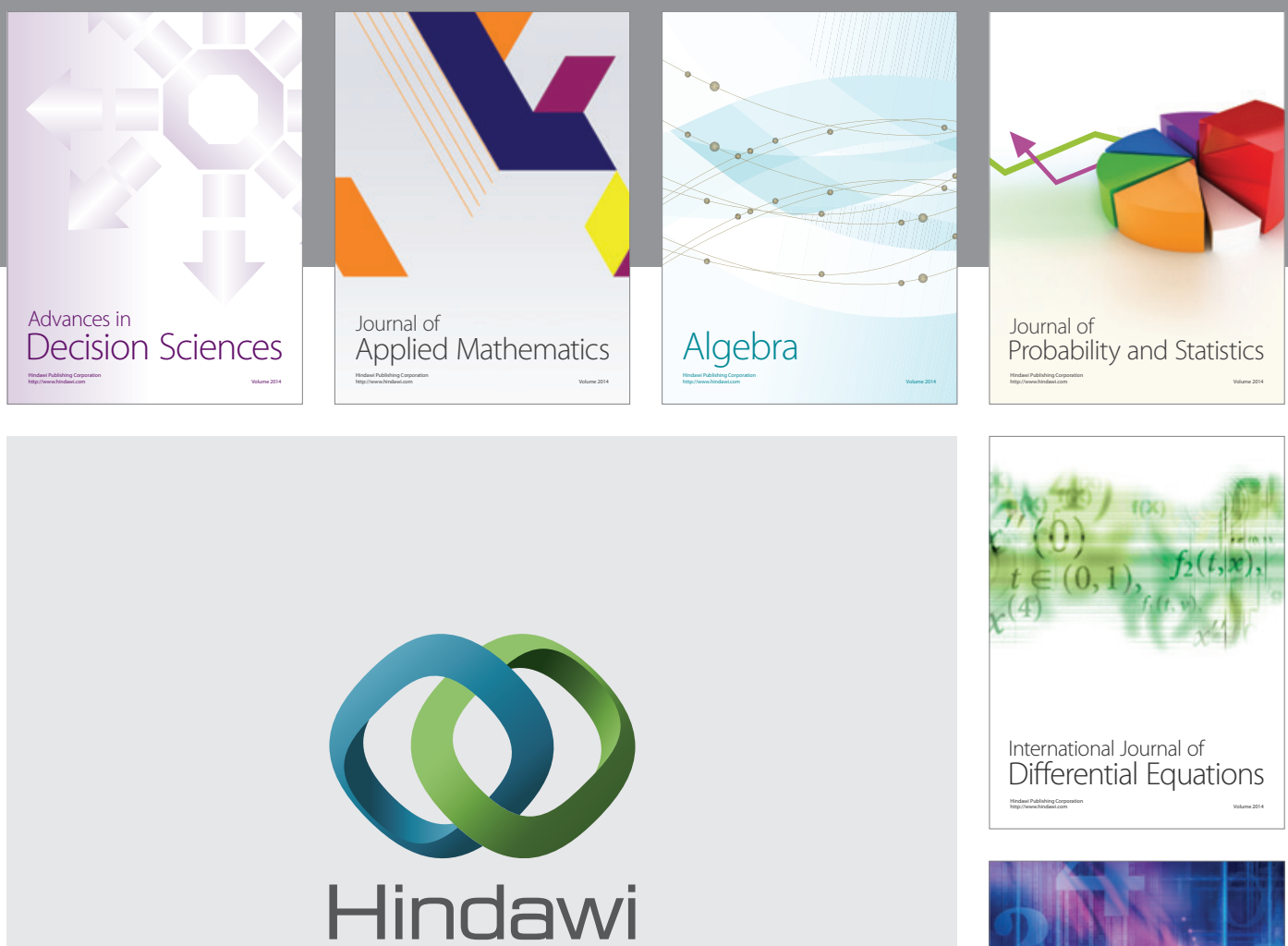

Submit your manuscripts at http://www.hindawi.com
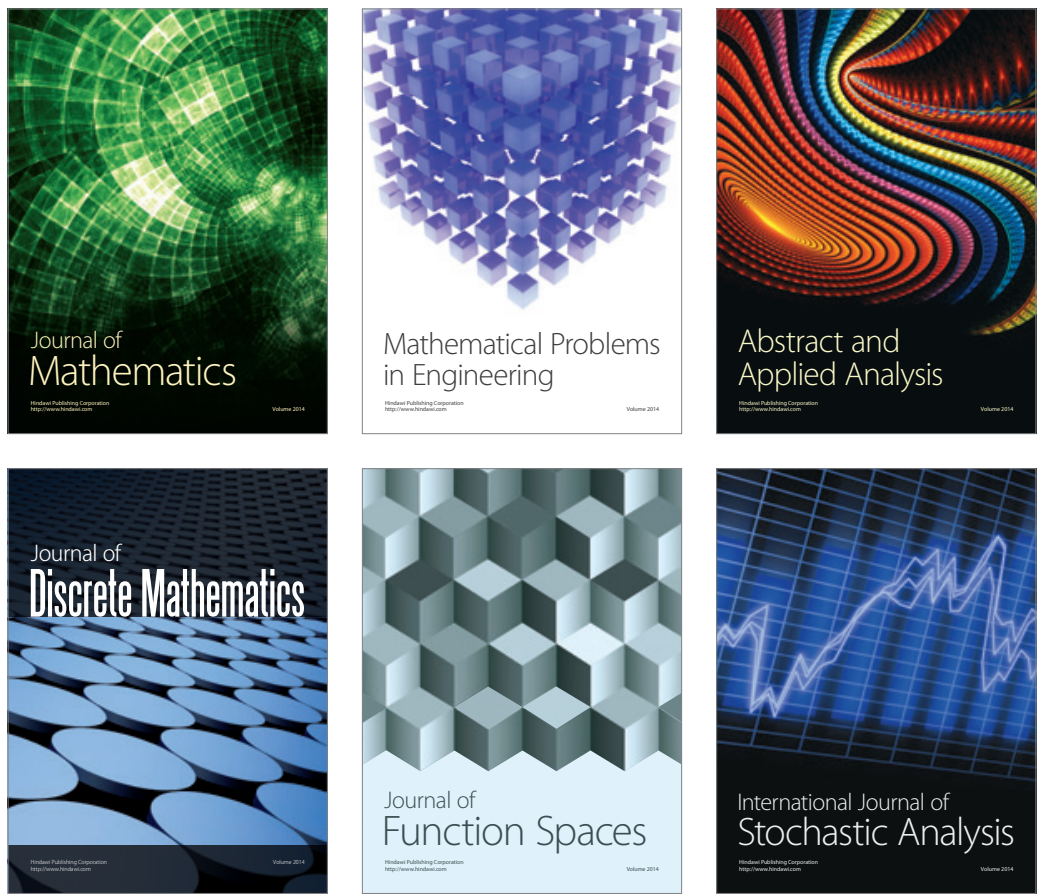

Journal of

Function Spaces

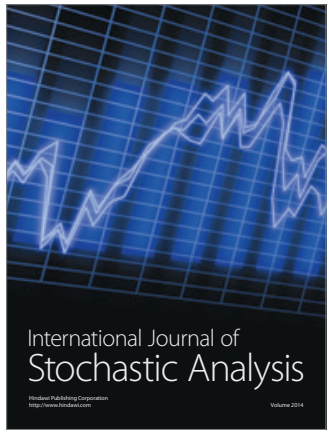

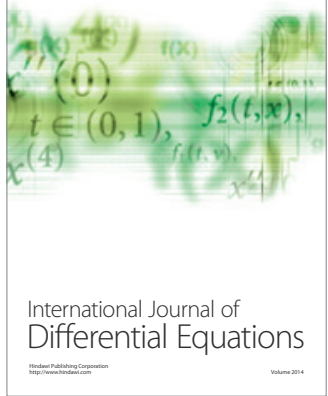
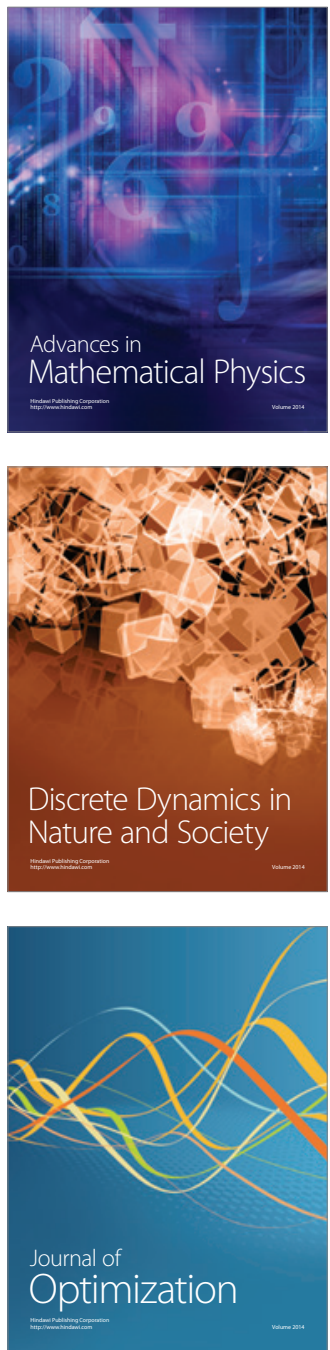